number must be extended, if possible to rooo, before the work of reduction is begun. May I ask for further aid in the matter? I shall be glad to send two or more schedules to anyone who will help to get a faithful record.

University College, London, W.C. Karl Pearson.

\section{Thermodynamics of Diffusion.}

Is applying the principles of thermodynamics to diffusion of gases, several pitfalls have to be guarded against.

In the first place, if we adopt the old definition of entropy in terms of integrals of the form $\int d Q / T$, we are almost certain to go wrong when we come to deal with diffusion. If we imagine diffusion to take place between two of the ideal "perfect gases" of our text-books at constant pressure, volume and temperature, and without gain or loss of heat, no quantity of the nature of $d Q$ appears to be associated with the phenomenon, and it is casy to rush to the conclusion that no change of entropy takes place. This danger is avoided if we adopt Mr. Swin burne.'s plan of defining entropy in terms of "waste" or unavailable energy relative to an assumed auxiliary medium. By "auxiliary medium" is here meant a medium at uniform temperature $T_{0}$ which can be used indefinitely as a refrigerator in thermodynamic operations, and any change in the amount of unavailable energy under such conditions, when divided by the temperature $T_{b}$, gives the corresponding change of entropy.

If this definition is adopted we see that the phenomenon of mixing the gases does not in itself suffice to determine the changes of entropy associated with it. The matter can only be decided by an appeal to experience as to the means whereby the gases can be separated or mixed reversibly. The case of an ideal "perfect gas" forms no exception to this statement.

The proper inference is, not that the diffusion involves no change of entropy, but that the change of entropy, if it exist, cannot be expressed as a sum of differentials of the form $d Q / T$

The second pitfall occurs when we take the well-known expression for the entropy of a perfect gas in terms of pressure (or volume) and temperature, and try by this means to connect the entropy of the mixture with the entropies of the components. Where we are likely to get into trouble is by ignoring the integration constants in the expressions for the entropy. There is no evidence from mere thermodynamic reasoning that the constant does not change in the process of diffusion. All we can infer is that the change of entropy associated with the mixing of gases at uniform pressure and temperature is constant, i.e. independent of pressure and temperature.

To sum up, then, even when we have defined an ideal perfect gas in the ordinary way, and assumed the property that two such gases can mix in a closed vessel without change of pressure and temperature, thermodynamical considerations still give us no information whatever as to the change of entropy accompanying diffusion, and on this point a further appeal to experience is necessary.

This amounts to saying that our definition of perfect gases is still incomplete. What further property shall we assume in order to complete it? If we regard a "perfect gas" as a mere invention on paper, the most useful plan is to take some simple property which is approximately satisfied in the case of actual gases and assume that this property is accurately satisfied by our perfect gases. Now, actual gases may be separated and re-mixed either by diffusion through a membrane or by liquefying, or, if preferred, freezing one of the constituents.

Taking either of these processes, and making suitable assumptions which would render that process perfectly reversible, we are led to the inference that the whole entropy of a mixture of perfect gases should be taken to be equal to the sum of the whole entropies of its components at the same temperature and partial pressure, i.e. if each component occupied the same volume as the final mixture.

According to this view, when diffusion takes place at No. I9I 5 , VOL. 74$]$ constant temperature and pressure, there is a gain of entropy and a loss of available energy equal in amount to that which. would be incurred if each of the constituents were to expand by rushing into a vacuum until it occupied the same volume as the final mixture.

There is another way of partially separating the constituents of a gas mixture. If the mixture be introduced into a field of force such as that due to the earth's attraction, or if we imagine it to be whirled in a centrifuge, the denser gases will predominate in the lower parts of the atmosphere or where the potential is greatest, and the lighter gases will predominate in the upper regions or where the potential is least. In this case the partial separation is effected at the expense of work done by the field of force.

This note does not purport to deal in full detail with the thermodynamics of diffusion, but merely to direct attention to certain points which are easily overlooked. One of the most important of these points is that the possibility of producing mechanical work by the diffusion of gases through a membrane at constant temperature is not necessarily inconsistent with the principles of thermodynamics or the ordinary definitions of a perfect gas.

If any physicist should claim to have discovered Maxwell's demons in connection with the diffusion of gases, the first questions we should ask him are :--

(1) Can he, without the performance of external work, separate the gases in a mixture in such a way that the temperature is the same at the end as at the beginning, and the separated constituents each occupy volumes smaller than that of the original mixture?

(2) Can he obtain external work by the mixing of two gases without change of temperature if the initial volume of each gas is not less than the final volume of the mixture?

(3) Are his claims based on new experimental evidence? G. H. Bryan.

\section{Early Meteors of the Perseid Shower.}

THe moon being new on July $2 \mathrm{I}$ this year renders the conditions favourable for observing the earlier members of the great Perseid display. A few of these are usually visible on July $I_{5}$, and probably just before that night, and it would be interesting if multiple observations of supposed Perseids could be obtained so that their radiants might be definitely assigned without the risk of error.

A single record of a meteor-flight only permits an assumption to be made as to the apparent radiant, and mistakes frequently result. For example, if a streakleaving meteor, seen at the July-August epoch, happens to be directed from the northern part of Perseus it wil! certainly be attributed to the Perseid swarm, though it may quite possibly have had its origin in a different shower from Cassiopeia, Andromeda, Aries, Camelopardus or Auriga. To avoid such errors of allocation it is proposed to maintain simultaneous watches this rear between July I5 and 28 from ro to $\mathrm{J} 2 \mathrm{p} . \mathrm{m}$, and the writer would be glad to hear particulars of any observations for comparison with similar results obtained at Bristol.

The mean height of the Perseid meteors has already been satisfactorily deduced, but it seems desirable further to investigate the position and motion of the radiant, especially during the last half of July. Such meteors as appear amongst the stars of Perseus or bordering constellations are the best for indicating the exact place of the radiant, and bright meteors should always be carefully registered. as they are very likely to have been noticed elsewhere. The centre of radiation travels from near $\phi$ Andromedze at the middle of July to a few degrees south of the starcluster at $\chi$ Persei at the end, the ephemeris places (Monthly Notices, Ixii., r69) being as under:-

Date R.A. Dec. 1 Date R.A. Dec.

$\begin{array}{lllllllll}\text { July } 15 & \ldots & \ldots & 15 \cdot 3+48 \cdot 9 & \text { July } 25 & \ldots & \ldots & 24^{\circ} 9+52^{\circ} \cdot 5\end{array}$

\begin{tabular}{lllllllllll}
, & 17 & $\ldots$ & $\ldots$ & I $7 \cdot \mathrm{I}+49 \cdot 7$ &,, & 27 & $\ldots$ & $\ldots$ & 27 \\
\hline & 1 & $+53 \cdot 2$
\end{tabular}

$\begin{array}{lllllllllll}, & 19 & \ldots & \ldots & 18 \cdot 0+50 \cdot 5 & , & 29 & \ldots & \ldots & 29 \cdot 3+53 \cdot 8\end{array}$

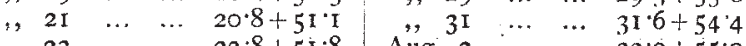

$\begin{array}{llllllllll},, & 23 & \ldots & \ldots & 22 & 8+51 \cdot 8 & \text { Aug. } 2 & \ldots & \ldots & 33 \cdot 9+55 \cdot 0\end{array}$

Bishopston, Bristol. 